\title{
An investigation of ambiguous-cue learning in pigeons
}

\author{
GEOFFREY HALL \\ University of York, York Y01 SDD, England
}

\begin{abstract}
Two experiments demonstrated that pigeons can solve a simultaneous discrimination in which on half the trials the positive and an ambiguous cue (A) are presented and on half the trials choice is between $\mathrm{A}$ and the negative stimulus. In Experiment 1, where a relatively nondistinctive A cue was used, performance on the former type of trial was superior to that shown on the latter. In Experiment 2, where a distinctive A cue was provided, this pattern of results was reversed. These findings are interpreted in terms of an approach-avoidance explanation first proposed by Leary (1958). Experiment 3 tested and confirmed a central prediction of this explanation by showing that in an orthodox simultaneous discrimination, occasional reinforcements of the negative stimulus produce less accurate performance than do nonreinforcements of the positive.
\end{abstract}

Leary (1958) trained rhesus monkeys on a simultaneous discrimination in which three stimuli were used. On half the trials, the subjects had to choose between the positive stimulus $(P)$ and a nonrewarded stimulus (A); on the remaining trials, choice was between the negative stimulus $(\mathrm{N})$ and a rewarded stimulus, which again was stimulus $\mathrm{A}$. This problem has been called an ambiguous-cue problem, since the value of cue $A$ depends upon the stimulus with which it is paired. The monkeys solved the problem in that they developed a preference for the rewarded stimulus on both types of trial. Performance was, however, better on NA trials than on PA trials, a result which may be abbreviated NA $>$ PA. This result has been found in several other experiments using primate subjects (Boyer \& Polidora, 1972; Boyer, Polidora, Fletcher, \& Woodruff, 1966; Fletcher \& Garske, 1972; Fletcher, Grogg, \& Garske, 1968).

These results stand in marked contrast to those produced by a number of other experiments on ambiguous-cue learning in primates. Fletcher and Bordow (1965) and Thompson (1954) found performance to be better on PA trials than on NA trials (PA>NA); Boyer and Polidora (1972) and Boyer et al. (1966) also report experiments in which this pattern of results was found. The chief feature distinguishing these experiments from those producing the NA $>$ PA result lies in the nature of the stimuli used. In Leary's experiment, for example, the stimuli were the "junk objects" often used in the Wisconsin General Test Apparatus. Thompson's (1954) experiment, on the

This work was supported by a grant from the U.K. Science Research Council. I thank E. Macphail and S. Channell for their comments. Requests for reprints may be sent to $G$. Hall, Department of Psychology, University of York, Heslington, York Y01 5DD, England. other hand, gave subjects a choice on each trial between two foodwells, next to one of which was a stimulus plaque, either $\mathrm{P}$ or $\mathrm{N}$, stimulus $\mathrm{A}$ being simply the absence of a stimulus plaque. The other experiments that produced the PA $>$ NA result used as stimuli the white lids covering the foodwells; the $\mathrm{P}$ and $\mathrm{N}$ stimuli were distinctively striped and colored but the lid serving as the A stimulus was left blank. In general, those experiments which used a separate and distinctive A stimulus produced the NA $>$ PA result; those that did not produced PA $>$ NA. This generalization has been tested by Boyer and Polidora (1972) and Boyer et al. (1966). These experiments first demonstrated the PA $>$ NA result with a stimulus set lacking a distinctive A stimulus, and then went on to show that NA $>$ PA is found when such a stimulus is provided.

The aims of the experiments reported here are: first, to show that pigeons can solve what seems at first sight to be a complex discrimination problem (Richards, 1973, has provided a preliminary demonstration of this); second, to try to generate in pigeons both the PA $>N A$ result and the NA $>$ PA result by manipulating appropriately the nature of the stimuli; and third, to provide an experimental test of the most popular explanation for these findings, an explanation which accounts for performance in this relatively complex situation in terms of simple conditioning principles (Berch, 1974; Leary, 1958).

\section{EXPERIMENT 1}

In this experiment, pigeons were trained on an ambiguous-cue problem with stimuli that, it was thought, would produce the PA $>$ NA result. A plain red field was used as the ambiguous cue; white stripes differing in orientation were superimposed on this red background to produce the $\mathbf{P}$ and $\mathbf{N}$ stimuli. The 
red background therefore bore the same relationship to the features distinguishing the $\mathrm{P}$ and $\mathrm{N}$ stimuli as did the white lid of the foodwell in the experiments with monkeys.

\section{Method}

Subjects and Apparatus. The subjects were seven pigeons which had previously been used in a study of free-operant discrimination learning. They had been autoshaped to respond to a key illuminated with white light and had formed a discrimination between a steady and a flashing houselight. No houselight was used in the present study. The birds were maintained throughout at $80 \%$ of their freefeeding weights. They were trained in a pigeon apparatus on one wall of which were three response keys, each $2 \mathrm{~cm}$ in diameter. The central key, positioned above a grain feeder and $20 \mathrm{~cm}$ from the floor, could be lit from behind with white light. The side keys were at the same level and were $8 \mathrm{~cm}$ (center to center) from the central key. Behind each side key was an in-line projector which could dis play a red field (the $R$ stimulus) or the red field with a single white line $(2.0 \times 0.2 \mathrm{~cm})$ superimposed. Lines running horizontally $(H)$, vertically (V), and the 45-deg oblique $(O)$ were used. This apparatus was mounted inside a sound-attenuating chamber.

Procedure. The birds were given daily sessions of 60 trials. The interval between trials was always $20 \mathrm{sec}$, and reinforcement consisted of access to grain for $4 \mathrm{sec}$. On the first day of pretraining, each trial consisted of the presentation of the white center key; a single response to this key turned off the light and produced reinforcement. On the next day, the subjects were pretrained to respond to the side keys. Each trial again began with the illumination of the center key; a peck to this key produced a stimulus on one of the side keys, and a response to this side key turned off the stimulus and was followed by reinforcement. Responses to unlit keys were without programmed consequences. The stimulus displayed on the side key was $\mathrm{R}, \mathrm{H}$, or $\mathrm{V}$. Each was presented 20 times and appeared equally often on the left and on the right. The scheduling of the stimuli was determined by modified Gellerman sequences (Fellows, 1967).

There followed a test session designed to assess the extent to which each of these three stimuli was preferred with respect to some other. On 20 trials, therefore, the birds were given a choice between $\mathrm{H}$ and the $\mathrm{O}$ stimulus, on 20 trials between $\mathrm{V}$ and $\mathrm{O}$, and on the remaining trials between $R$ and $O$. These trials were organized in the same general way as previously, except that two side keys were presented simultaneously and a response to either turned off both and resulted in reinforcement.

The next 10 sessions comprised training on the ambiguous-cue problem. For all the subjects $R$ was cue $A, H$ was cue $P$, and $V$ was cue N. Half of each day's trials were PA and half were NA; within each set of trials, $A$ appeared equally often on the left and on the right. A response to the appropriate side key (P, or A on NA trials) produced reinforcement, but a response to the other key simply produced the 20 -sec dark interval. Response to either side key turned off both key lights.

\section{Results and Discussion}

On the first session of training on the ambiguous-cue problem, the mean score for the group was $50.4 \%$ correct responses; by the last session, this score had risen to $59 \%$, suggesting that learning proceeded only slowly. These overall means obscure a marked difference in performance on the two types of trial. This is shown in Figure 1, which makes it apparent that PA performance was superior to NA performance throughout. An analysis of variance carried out on the data shown in the figure produced a significant

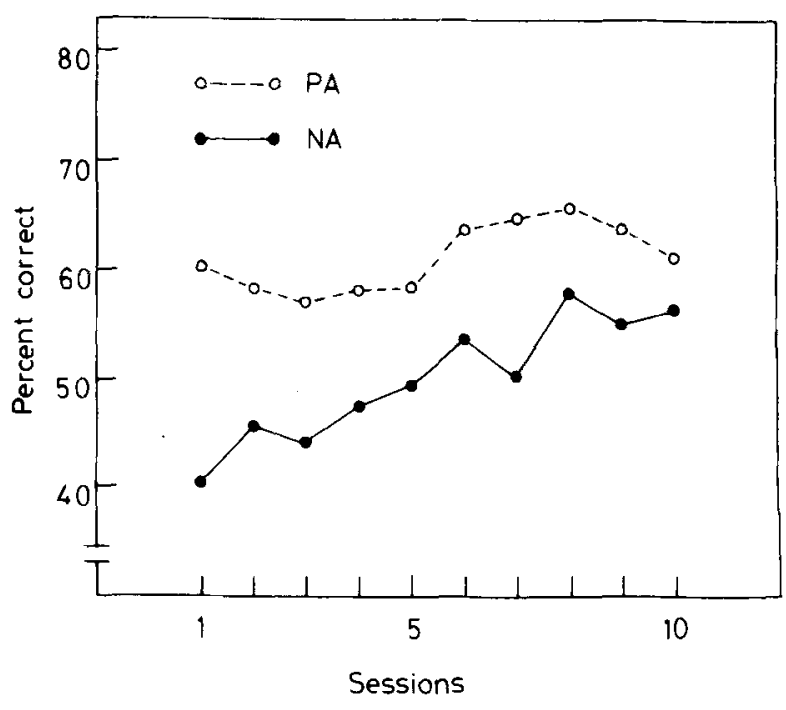

Figure 1. Mean percent correct on the ambiguous-cue problem in Experiment 1. PA, trials with positive and ambiguous cues; NA, trials with negative and ambiguous cues.

effect of trial type $[F(1,6)=9.28, p<.05]$ and a marginally significant effect of sessions $[F(9,54)=$ $2.01, \mathrm{p}<.1]$. The interaction between these two factors was not significant. It is also apparent that the main effect of training is to raise the level of performance on the NA trials which started at below the chance level $(50 \%)$. On the first session of training, four of the subjects scored below $50 \%$ on NA trials. In contrast, all but one of the subjects scored above $50 \%$ on PA trials during this session.

This preference for stimuli including the white line (i.e., the $\mathrm{H}$ and $\mathrm{V}$ stimuli) over the red stimulus was not apparent on the test session given before training began. On the trials where choice lay between $R$ and $O$, $R$ was chosen on $46 \%$ of occasions. This group mean score is representative of the performance of the individual subjects. Three showed no preference on these trials, and of the remainder the most extreme preference was shown by a subject who chose $R$ rather than $\mathrm{O}$ on 7 of the 20 trials with these stimuli. The other test trials also failed to reveal any marked preferences; $H$ was chosen overall on $48 \%$ of the $H$ vs. O trials, and $\mathrm{V}$ on $51 \%$ of the $\mathrm{V}$ vs. $\mathrm{O}$ trials. The failure to find a preference for the striped stimulus over the plain red stimulus on the test session (given that such a preference was present on the first training session) may mean either that the test session provided an insensitive measure or that the preference developed very rapidly during the first training session and was therefore not present during the test session. At any rate, it was the existence of this preference during training that produced the PA $>$ NA result. The extent to which this and other $\mathrm{PA}>\mathrm{NA}$ results can be fully 
explained in terms of stimulus preference effects will be discussed after the next two experiments have been described.

\section{EXPERIMENT 2}

The stimuli used in Experiment 1 were intended to parallel those used in experiments with monkeys which produced PA $>$ NA. In the present experiment, a distinctive A stimulus was supplied in the hope of reversing the outcome of the previous study and producing NA $>$ PA. Horizontal and vertical stripes were again used as the $\mathrm{P}$ and $\mathrm{N}$ stimuli, but there was no red background and a white cross on a dark background was used as the A stimulus.

\section{Method}

The subjects were four pigeons that had previously learned a free-operant successive discrimination between key lights differing in color. After pretraining to respond to the white center key and to $\mathrm{H}, \mathrm{V}$, and the cross $(\mathrm{X})$ on the side keys, they received 12 sessions of training on the ambiguous-cue problem. For all subjects, $H$ was the positive, $V$ the negative, and $x$ the ambiguous cue. There were no test sessions. The $x$ stimulus was produced by simultaneous presentation of the 45- and 135-deg oblique lines. Procedural details not specified here were the same as in Experiment 1 .

\section{Results and Discussion}

With only four subjects, it is appropriate to present the performance of each on the two types of trial. Figure 2 shows that all subjects rose above chance level on both the PA and the NA trials. On the NA trials, scores started at about $50 \%$ and rose (rapidly for three birds) to $90 \%$ or better. Performance on the PA trials was less accurate; it started below $50 \%$ for three subjects and never reached the high level shown on NA trials. These results show that pigeons can solve the ambiguous cue problem and produce the NA $>$ PA effect. They confirm the findings of Richards (1973), who used a training procedure very like that used here and green, red, and orange key lights as the stimuli.

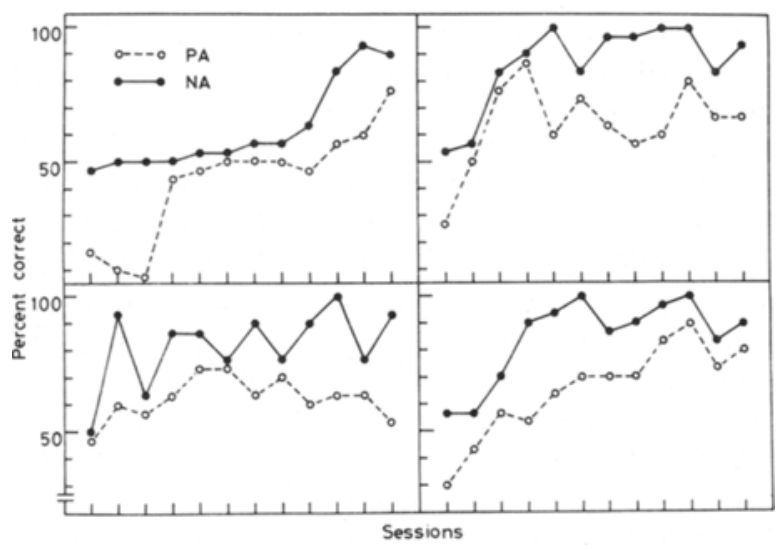

Figure 2. Percent correct for individual subjects in Experiment 2. PA, trials with positive and ambiguous cues; NA, trials with negative and ambiguous cues.
An explanation for results of this sort (i.e., those showing NA $>$ PA) can be developed from a relatively simple set of assumptions about approach and avoidance responses (see Berch, 1974; Leary, 1958). If we assume that a rewarded response to a stimulus produces a tendency to approach (and in this case peek at) that stimulus and that a nonrewarded response produces an avoidance tendency, it follows that subjects will come to approach $P$ and to avoid $N$. If the subjects receive a roughly equal number of rewards and nonrewards for responding to $\mathrm{A}$, it seems likely that the overall result will be an approach tendency. The effects of the rewards may be assumed to outweigh those of the nonrewardsanimals will, after all, learn new responses and maintain performance under a $50 \%$ partial reinforcement schedule. Thus, on NA trials, the animal must choose between a negative stimulus and one that it has some tendency to approach (stimulus A); performance should therefore be quite good. Performance on PA trials will be inferior, since here a tendency to approach A will be a handicap. The animal is, in effect, faced on these trials with a choice not between positive and negative but between positive and more positive.

If we are to accept this explanation, it will clearly have to be extended or modified to encompass those experiments that produce the PA $>$ NA result. But before considering this issue, it seemed worthwhile as a first step to try to subject the central assumption of the explanation to experimental test.

\section{EXPERIMENT 3}

This experiment was designed to test the critical assumption that underlies the approach-avoidance explanation of the NA $>$ PA result: the assumption that the positive effects of rewards in discrimination learning will outweight the effects produced by the same number of nonrewards. The procedure adopted was to train birds on an orthodox (P vs. $\mathrm{N}$ ) simultaneous discrimination until they were reliably responding to stimulus $\mathrm{P}$ rather than stimulus $\mathrm{N}$. They were then given training with just one of the stimuli. Birds in one group (Group P) were allowed to respond to the $P$ stimulus, but responses were not rewarded. Birds in Group $\mathrm{N}$ were required to respond to the $\mathrm{N}$ stimulus for the same number of trials, and responses to this stimulus were rewarded. The effects of these procedures on performance of the simultaneous, P-N, discrimination was noted. It was anticipated that nonrewards for responding to $P$ in Group $P$ would detract little from the approach tendency governed by this stimulus and that accurate performance would thus be maintained by this group on the P-N task. On the other hand, rewarded responses to the $\mathbf{N}$ stimulus should, according to the approach-avoidance theory, markedly increase the approach tendency elicited by this stimulus in Group P, rendering accurate performance difficult on the P-N task. 


\section{Method}

The apparatus was that used in the previous experiment. The subjects were 12 pigeons, again maintained at $80 \%$ of their freefeeding weights. Since these birds had previously learned a successive free-operant discrimination between lines differing in orientation, colored key lights were used as the stimuli. These were plain red and orange fields. Richards (1973) has shown that the NA $>$ PA result is found when stimuli of this sort are used in an ambiguous-cue problem.

Preliminary training was carried out as in the previous experiments, except that on the 2nd day red and orange were displayed on the side keys. All animals then learned a simultaneous discrimination between red and orange with red as the positive stimulus. Red and orange were presented on all trials, the positive appearing equally often on the left and on the right. In other respects, the training procedure was identical to that used in Experiments 1 and 2. Each subject was trained to a criterion of 10 successive correct responses, at which point the session was terminated automatically. Three further (overtraining) sessions, each of 60 trials, were given.

The subjects were then divided into two groups. Both received eight further sessions with 30 of their 60 daily trials being on the red-orange discrimination. The groups differed in the treatment they received on the remaining trials of the session. Group $P$ received 30 trials, intermixed with the red-orange trials, on which just the red stimulus was presented. It was shown 15 times on the left and 15 times on the right, the other side key remaining unlit. Response to the red stimulus was not rewarded. Group $N$ received similar, intermixed, single-stimulus trials, on which only the orange stimulus was presented. Response to this stimulus resulted in reward. Pilot work has shown that pigeons that have been trained on a simultaneous discrimination will always peck a single illuminated side key and that they will do so (although often with a lengthened latency) even when the lit key has previously been established as a negative stimulus. Throughout training, responses to unlit keys were without programmed consequences.

It will be noted that this procedure of intermixing red-orange and single-stimulus trials makes the present experiment a close parallel to the ambiguous-cue problem itself. For Group $P$, nonrewarded experience with the red stimulus on the single-stimulus trials turns the red-orange discrimination into a task requiring choice between a negative stimulus and a stimulus that is sometimes rewarded and sometimes not. In this way, the red-orange task becomes equivalent to an NA subproblem. In an analogous fashion, the red-orange task becomes the equivalent of a PA subproblem for Group N.

\section{Results and Discussion}

Figure 3 shows the performance of the two groups on the overtraining sessions on red-orange and their performance on this problem after the introduction of single stimulus trials. The groups did not differ during overtraining on red-orange. An analysis of variance carried out on the overtraining data presented in the figure showed a significant effect of sessions $[F(2,20)=3.55, p<.05]$, but no significant effect of groups $[F(1,10)=3.05, p>.1]$ and no significant interaction between these two factors $(F<1)$. Group $P$ maintained its level of performance when singlestimulus trials were introduced, but Group $\mathrm{N}$ showed a marked decline in performance. Over the eight sessions of training in these conditions, the groups differed significantly $[F(1,10)=12.59, p<.01]$. There was a significant effect of sessions $[F(7,70)=5.06$, $p<.01]$, and the interaction between sessions and groups was also significant $[F(7,70)=4.42, p<.01]$.

The approach-avoidance account of ambiguous cue learning is supported by these results. Nonreward

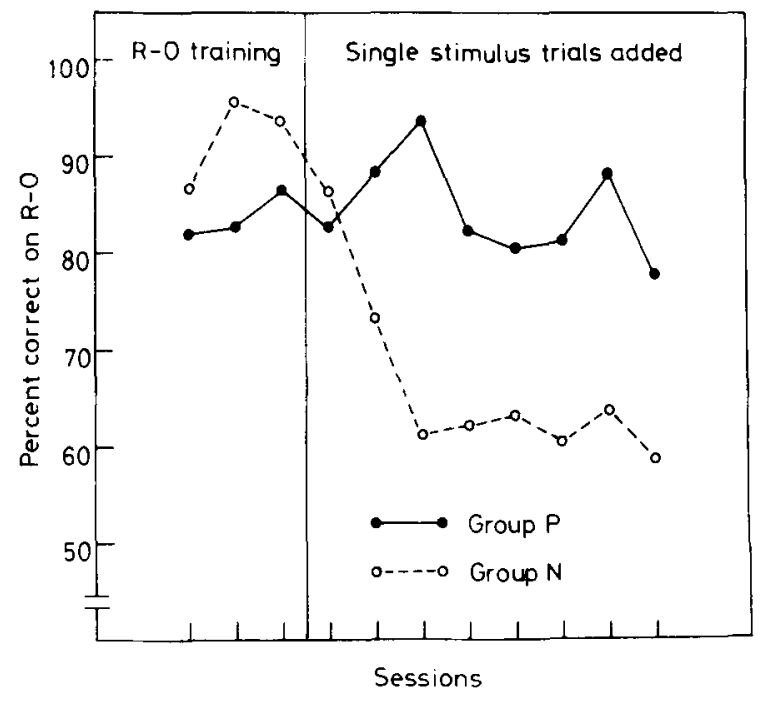

Figure 3. Mean percent correct on red-orange (R-O) discrimination. Group $P$ received added nonreinforced trials with the positive stimulus; Group N received added reinforced trials with the negative.

for reponse to the positive (which occurs, in this case, with roughly the same frequency as reward) does not disturb performance on a simultaneous discrimination. But equivalent reward for response to the negative does disturb performance. By this measure, therefore, reward proves to be a much more effective (in this case, disruptive) procedure than nonreward.

\section{GENERAL DISCUSSION}

The first two experiments establish that pigeons can solve an ambiguous-cue problem. They further show that PA performance is superior to NA performance when there is no distinctive $A$ stimulus, but that NA performance is superior to PA performance when a distinctive $A$ stimulus is available. The results of the third experiment lend support to an account of ambiguous-cue learning that predicts the NA $>$ PA result. It remains, therefore, to determine if this account can be extended to deal with experiments producing the PA $>$ NA result such as Experiment 1 of this report.

One possibility requires the approach-avoidance theory to be modified hardly at all. If we adopt the plausible assumption that primates have a tendency to respond to a distinctive stimulus object rather than to an unmarked foodwell, it follows that PA performance would be helped and NA performance hindered. This might be enough to outweigh the factors that tend to produce the NA $>$ PA result. Boyer et al. (1966), who found a PA $>$ NA result, tested some subjects on $P A$ alone and others on NA alone before transferring them to the ambiguous-cue problem itself. They found that although the PA problem was learned readily, the NA problem was not. Thus, a natural 
preference for a cued over a noncued foodwell seems enough to explain their results.

Other experiments producing PA $>$ NA cannot be immediately explained in this way. Thompson (1954) tested stimulus preferences by training a group of subjects on a discrimination between the presence and absence of a stimulus card. Those trained with the empty stimulus holder as the positive learned as readily as those given a stimulus card as the positive. Fletcher and Bordow (1965) included a similar test, and in this case, too, no preference was found. To explain their findings, Fletcher and Bordow put forward what may be viewed as a modified version of the stimulus preference account just described; they suggest that, as a result of ambiguous-cue training itself, the subjects may develop a preference for cued over noncued foodwells which outweighs the factors promoting the NA>PA result. Their suggestion uses the notion of stimulus generalization.

The NA $>$ PA result is found when three separate and different stimuli are used - junk objects in Leary's (1958) experiment; and different patterns of stripes in the present Experiment 2. With these stimuli there will be little generalization, or at least there will be no more generalization from $\mathrm{P}$ to $\mathrm{N}$ than from $\mathrm{P}$ to A. But with the stimuli used by Fletcher and Bordow themselves, by Thompson (1954), and in the present Experiment 1, we might expect differential generalization to occur. There will be generalization between $\mathrm{P}$ and $\mathrm{N}$, which are similar, but not between $\mathrm{A}$ and the other stimuli. If we now make our usual assumption about the preeminence of the approach tendency produced by reward, we can expect the $\mathrm{N}$ stimulus to acquire a greater approach tendency by generalization than the avoidance tendency acquired by the $P$ stimulus by generalization from $\mathbf{N}$. It could thus occur that the difference in approach strength between $P$ and $A$ would be greater than that between $\mathbf{N}$ and $A$. In this way, we can account for the occurrence of the PA $>$ NA result with certain stimuli without departing from the principles used to explain the NA $>$ PA result.
The ambiguous-cue problem seems at first sight to be a complex one that might tax the abilities even of primates. It is of interest, therefore, that pigeons can solve this problem and that an explanation can be derived, both for their performance and that of the primates, from a relatively simple theory of discrimination learning that departs very little from the principles suggested by Spence $(1936,1937)$.

\section{REFERENCES}

BERCH, D. B. A theoretical analysis of the PAN ambiguouscue problem. Learning and Motivation, 1974, 5, 135-148.

Boyer, W. N., \& Polidora, V. J. An analysis of the solution of PAN ambiguous-cue problems by rhesus monkeys. Learning and Motivation, 1972, 3, 325-333.

Boyer, W. N., Polidora, V. J., Fletcher, H. J., \& Woodruff, B. Monkeys' performance on ambiguous-cue problems, Perceptual and Motor Skills, 1966, 22, 883-888.

Fellows, B. Chance stimulus sequences for discrimination tasks. Psychological Bulletin, 1967, 67, 87-92.

Fletcher, H. J., \& Bordow, A. M. Monkey's solution of an ambiguous-cue problem. Perceptual and Motor Skills, 1965, 21, 115-119.

Fletcher, H. J., \& Garske, J. P. Response competition in monkeys' solution of PAN ambiguous-cue problems. Learning and Motivation, 1972, 3, 334-340.

Fletcher, H. J., Grogg, T. M., \& Garske, J. P. Ambiguouscue problem performance of children, retardates, and monkeys. Journal of Comparative and Physiological Psychology, 1968, 66, 477-482.

LEARY, R. W. The learning of ambiguous cue problems by monkeys. American Journal of Psychology, 1958, 71, 718-724.

Richards, R. W. Performance of the pigeon on the ambiguouscue problem. Bulletin of the Psychonomic Society, 1973, 1, 445-447.

SPENCE, K. W. The nature of discrimination learning in animals. Psychological Review, 1936, 43, 427-449.

Spence, K. W. The differential response in animals to stimuli varying within a single dimension. Psychological Review, 1937, 44, 430-444.

Thомpson, R. Approach versus avoidance in an ambiguous-cue discrimination problem in chimpanzees. Journal of Comparative and Physiological Psychology, 1954, 47, 133-135.

(Received for publication June 28, 1979; revision accepted October $30,1979$. 